\title{
The Permian Clayey Sediments Genesis by XRF Data on the Reference Section (Volga River, Russia)
}

\author{
R.R. Khaziev*, G.A. Krinari, N.G. Nurgalieva, G.A. Batalin and B.I. Gareev \\ Institute of Geology and Petroleum Technologies, Kazan Federal University, 420018 Russian Federation, Russia; \\ radmir361@mail.ru,krinari@ksu.ru, Nurgal07@mail.ru,bulat@gareev.net.georgii@batalin.com
}

\begin{abstract}
Background/Objectives: To study provenance and epigenetic transformation of clayey sediments, which were collected during the expedition in the summer of2013. Methods/Statistical analysis: Main method, which used in this work: $\mathrm{x}$-ray fluorescence analysis, which conducted on the spectrometer S2 RANGER (BRUKER). Preparing of sample for analysis included its crushing in mill for 5 minutes to a particle size of about 40-50 $\mu \mathrm{m}$; compaction with boric acid in a press with a force of $200 \mathrm{kN}$ to get a tablet with a very smooth surface. Findings: For the first time in the present work, by using data of the chemical composition and construction of petrochemical diagrams established: 1) geodynamic settings of sedimentation Tatarian age rocks (passive continental margin); 2) dominant source of demolition - mafic rocks;3) identified epigenetic changes clay rocks (low temperature smectite illitization). The obtained data are consistent with the results of previous researchers. The same conclusions have previous researchers in the results of macro- description of outcrop; description of microsections clayey rocks; electronic microscopy, x-ray structural analysis. It should be noted, that sampling section in the Monastirsky ravine made with a high detail step: totally allocated 360 layers of all five suites, 183 sample were analyzed. Application/Improvements: Analysis of petrochemical diagrams, by using data of chemical composition, proved to be a reliable method of complex reconstruction provenance and geodynamic settings in the study outcrop of Monastirsky ravine.
\end{abstract}

Keywords: Clayey Rocks, Epigenetic Transformation, Permian Sediments, Smectite Illitization, X-ray Fluorescence

\section{Introduction}

In this paper, we consider elemental composition and origin of claystone and siltstone sediments of the Urzhumian and the Tatarian ages within the section of Monastirsky ravine (Volga river, Russia), which is a key section for the studied sediments. The composition of the outcrops consists of five suites allocated by Forsch and Manuilenko in 1938. This stratigraphic division was further developed in the works ${ }^{1-3}$.In early stratigraphic schemes, all the five suites were considered as sediments of the Tatarian stage. Currently, the first, the second and a low part of the third suites are included in the Urzhumian stage; other parts of the third suite and the fourth suite are assigned to the Severodvinian stage; the fifth one is connected to the Vyatkian stage ${ }^{4,5}$.

In this work, the phenomenon of smectite transformation process was considered. This process frequently occurs in soils due to the interaction of clay and organic matter ${ }^{5}$. In these conditions, the transition from smectite to illite can flow through both intermediate mixed-layer minerals or by direct illitization at any temperature and certain Eh values that depend on the type of biotic assemblage at the stage of lithification and further diagenesis ${ }^{6}$.

It should be noted that biochemical processes may be significant in accumulated mud in arid environments, irrespective of the weathering crust. Thermodynamics, which specifies zoning of weathering crust, does not allow the presence of any metastable phase minerals, including mixed-layer minerals first and foremost ${ }^{7}$. The source of fine-grained sediment components may not be weathering crust, but overlying soil instead, even if they are completely washed out and transferred to the basin ${ }^{\circ}$. Further composition of clay minerals is primarily formed not by weathering processes, but by the $\mathrm{p}$ henomenon of smectite illitization.

${ }^{*}$ Author for correspondence 


\section{Object}

Monastirsky ravine is located in the Tetyushki district of the Republic of Tatarstan, $12 \mathrm{~km}$ away north of the town of Tetyushi (Figure 1). The construction of lithological column of the Urzhumian and Tatarian rocks in the ravine is based on the detailed description of the section, which is presented in ${ }^{9}$, as well as on the research of the collection of samples taken from the clayey sediments of the five suites during an expedition in 2013.

The first suite is exposed on the right side of the ravine, where a series of steep walls is formed. Its thickness is approximately 45 meters. The suite is represented by a rhythmic alternation of red clay, clay-carbonate and siltstone sediments interbedded with limestone and dolomite; there are bands of palygorskite in its upper part. In its lower part the suite is represented by a formation, which in most cases goes with gypsum with numerous bands of dolomite marl. There is also an alternation of clay-carbonate rocks interbedded with palygorskite (Figure 3). The top of the formation is composed mostly of clayey rocks. A characteristic feature of this suite is the absence of layers containing faunal remains.

The composition of rock-forming minerals and geochemical characteristics of the formation studied in 10-12 indicates that the lower part of rocks of the first suite (thickness up to $10 \mathrm{~m}$ ) was formed in the environments of isolated sub saline basin. While its "lifeless" in paleontological respect dolomitic and marl interlayers, as well as palygorskite interlayers, can be explained by transient increase in salinity of the basin, which complicated living ecosystems for biotic assemblages. However, by the end of the accumulation process of the first suite sediments salinity of the basin fell down and the suite became favorable for the development ofbiota, as it is evidenced by findings in the fossil sediments of crustaceans and fish scales ${ }^{\frac{13}{3}}$.

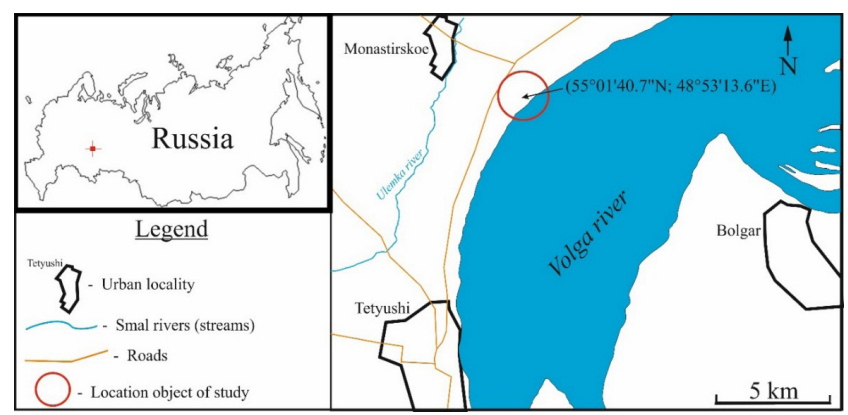

Figure 1. Overview map with the location of the object of study.
The second suite is exposed similarly to the first one in the right side of ravine, as well as in different parts of the thalweg of the ravine. Its thickness is 36 meters. Its lower boundary is carried out on the top of pinkish-gray dolomitic marl, which is connected to the first suite. A distinctive feature of the second suite is a higher content of carbonate rocks and clearly expressed recurrence, manifested in the occurrence of three clay-carbonate packs (at the bottom, middle and top of the suite) (Figures 2, 3),

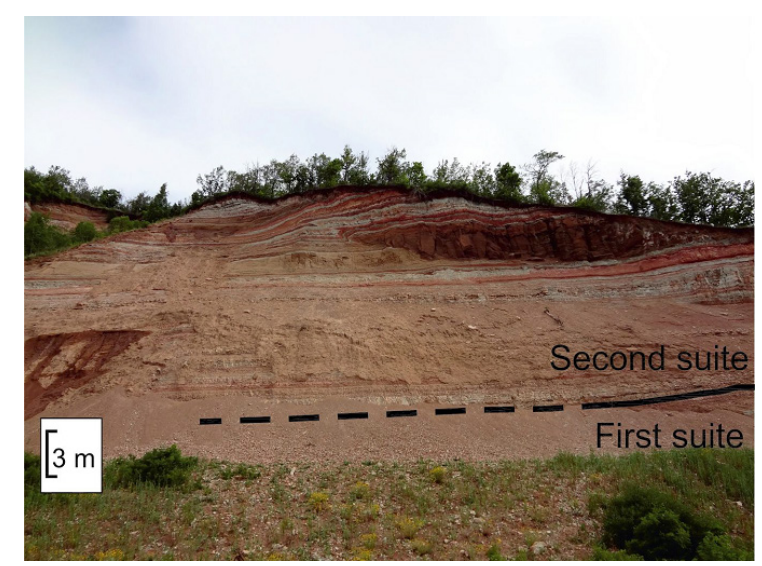

Figure 2. The Outcrop of the first and second suites on the left side of Monastirsky ravine.

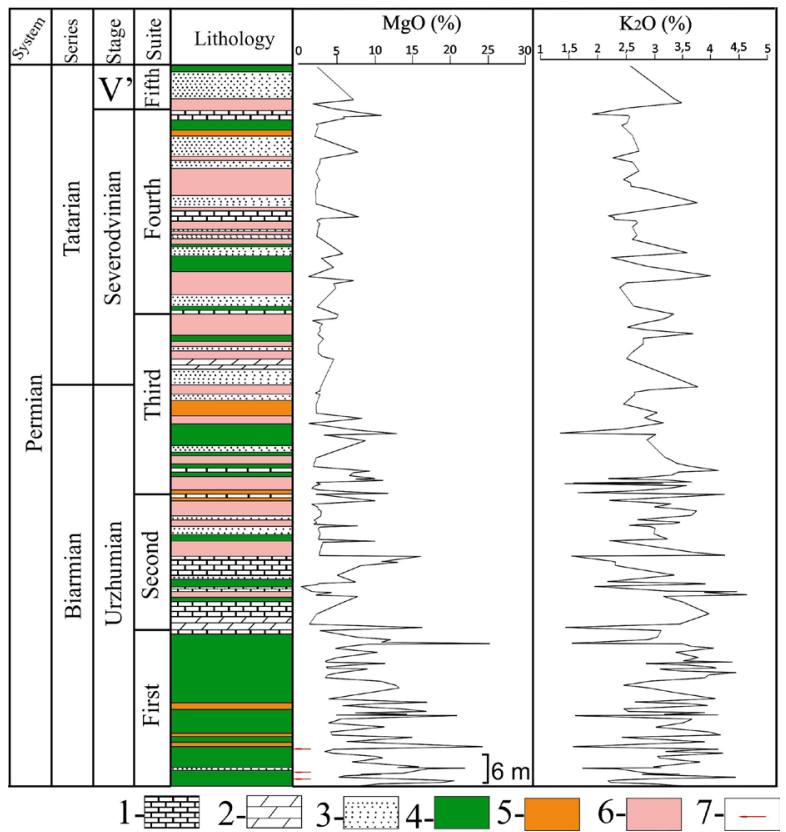

Figure 3. Stratigraphic succession of Monastyrsky ravine and chemical variations of $\mathrm{MgO}$ and $\mathrm{K}_{2} \mathrm{O}$. Legend: 1-limestones; 2-marls; 3-sandstones; 4-claystones; 5-siltstones; 6-paleosols; 7-palygorskite levels. (Note V’ in stratigraphic scheme - Vyatkian stage). 
which are clearly separated from each other by batches of sand and clay rocks. Packs of the second suite are clearly compared by faunal remains with the Isheevskaya suite of the outcrops of the Cheremushka ravine $e^{14}$, where the outcrop is represented by alternating brick-red clay, consisting of mixed-layer minerals with smectite; siltstone with flakes of mica and grains of plagioclase; dolomitic marl, containing illite-smectite phase; as well as fish residues in $7 \mathrm{~m}$ above the top. Changes in the composition of rocks suggest that they were formed in the sub saline sea basin, where as a result of salinization there occurred cyclic carbonate formation of precipitation alternated with clay-silty sediments, which were accumulating in the environment of desalination.

In ${ }^{15}$ Urzhumian sediments have been studied in the outcrop of Cheremushka by X-Ray Fluorescence analysis (XRF) and by electron paramagnetic resonance (EPR); there were outlined geochemical zones and cycles associated with the change of salinity regime and increasing dominance of continental sedimentary environments in arid climate environments.

The third suite is exposed in the upper right side of ravine. Thickness of the suite is about $36 \mathrm{~m}$. Its bottom is carried out on the top of the upper carbonate pack of the second suite which forms a "cliff-waterfall" in the thalweg of the ravine. A characteristic feature of the third suite is the predominance of sandstones and siltstones in its outcrop. Carbonate interlayers are insignificant, and their thickness is not great. The most common rocks are brownish-red clay and siltstone, interbedded with thick layers of tan cross-bedded sandstones, which form steep cliffs and slopes in the thalweg of the ravine. The suite is characterized by a distinct recurrence and is divided into five packs: the odd ones are of sand and clay; the even ones are of clay-carbonate. Petrographic composition of the rocks and ratio change of rock-forming minerals indicate that the rocks of the suite were formed in the environment of shallow brackish basin, occasionally desalinating due to the influx of substantial quantities of river waters from the adjacent land.

The fourth suite is exposed in the thalweg of the upper left side of the Monastirsky ravine, which is represented by red mudstones and siltstones, sandstones containing sand lenses and interlayers of carbonate rocks. Its thickness is $33 \mathrm{~m}$. The lower boundary of the suite is performed at the base by the gray marl and limestone. There are five packs: the odd ones are of clayey-carbonate rocks; the even ones are of sandy rocks. The mineral composition of clayey sediments varies from layer to layer resulting in a change in the quantitative content of montmorillonite and illitesmectite mixed associations. Clay, which is accumulated in the freshwater environment, is characterized by high content of montmorillonite. General patterns of change in the composition of rock-forming minerals indicate that rocks in the suite were formed in a basin with unstable saline regime. Thus, four suites are composed of freshwater, brackish and saline water sediments.

The fifth suite includes sandy and clayey packs that are exposed at the very edge of the upper left side. It is composed of yellowish-gray polymictic sandstones, including bands of reddish brown clay. The sandstones contain small layers of clay and marl.

\section{Methods}

Totally 183 samples from clayey layers were analysed. Chemical composition of rock-forming oxides is shown in Tables 1-5.

Table 1. Major element composition of clayey sediments first suite.

\begin{tabular}{|c|c|c|c|c|c|c|c|c|c|c|c|}
\hline Sample & Lithology & $\mathrm{SiO}_{2}(\%)$ & $\mathrm{Al}_{2} \mathrm{O}_{3}(\%)$ & $\mathrm{Fe}_{2} \mathrm{O}_{3}(\%)$ & $\mathrm{K}_{2} \mathrm{O}(\%)$ & $\mathrm{MgO}(\%)$ & $\mathrm{CaO}(\%)$ & $\mathrm{Na}_{2} \mathrm{O}(\%)$ & $\mathrm{TiO}_{2}(\%)$ & $\mathrm{P}_{2} \mathrm{O}_{5}(\%)$ & $\mathrm{Total}$ \\
\hline M01-1 & Clay & 57,26 & 17,29 & 6,32 & 3,57 & 10,13 & 3,55 & 0,54 & 0,8 & 0 & 99,46 \\
\hline M01-2 & Clay & 52,83 & 16,18 & 5,57 & 3,17 & 12,71 & 7,59 & 0,53 & 0,77 & 0 & 99,34 \\
\hline M01-3 & Clay & 37,15 & 11,57 & 4,37 & 2,31 & 20,86 & 21,6 & 0,58 & 0,64 & 0 & 99,09 \\
\hline M01-5 & Clay & 31,23 & 10,44 & 3,35 & 2,27 & 22,69 & 28,29 & 0,56 & 0,43 & 0 & 99,25 \\
\hline M01-6 & Clay breccia & 38,87 & 12,3 & 4,19 & 2,49 & 19,92 & 20,15 & 0,66 & 0,54 & 0 & 99,12 \\
\hline M01-7 & Clay & 52,32 & 16,7 & 5,9 & 3,23 & 12,61 & 7,46 & 0,45 & 0,67 & 0 & 99,35 \\
\hline M01-8 & Clay & 58,15 & 18,85 & 7,38 & 4,51 & 7,47 & 1,64 & 0,41 & 0,86 & 0,09 & 99,35 \\
\hline M01-9 & Clay & 60,53 & 17,9 & 5,62 & 3,86 & 8,16 & 2,19 & 0,49 & 0,8 & 0 & 99,55 \\
\hline M01-10 & Clay & 52,26 & 13,89 & 4,3 & 2,88 & 13,05 & 11,55 & 0,72 & 0,75 & 0 & 99,39 \\
\hline
\end{tabular}


The Permian Clayey Sediments Genesis by XRF Data on the Reference Section (Volga River, Russia)

\begin{tabular}{|c|c|c|c|c|c|c|c|c|c|c|c|}
\hline M01-11 & Clay & 56,89 & 16,98 & 6,27 & 3,53 & 10,41 & 3,97 & 0,53 & 0,86 & 0 & 99,44 \\
\hline M01-12 & Clay & 45,2 & 14,54 & 5,12 & 2,98 & 15,97 & 14,29 & 0,43 & 0,66 & 0 & 99,2 \\
\hline M01-15 & Clay & 45,48 & 11,95 & 4,02 & 2,46 & 19,18 & 15,6 & 0 & 0,61 & 0 & 99,3 \\
\hline M01-16 & Clay & 34,75 & 9,15 & 2,73 & 1,83 & 24,1 & 25,58 & 0,69 & 0,4 & 0 & 99,23 \\
\hline M01-17 & Clay & 47,81 & 14,09 & 4,56 & 3,21 & 15,26 & 13,43 & 0,44 & 0,53 & 0 & 99,33 \\
\hline M01-19 & Clay & 40,27 & 13,89 & 4,85 & 3,07 & 17,99 & 18,15 & 0,53 & 0,52 & 0 & 99,27 \\
\hline M01-21 & Clay & 57,72 & 18,68 & 6,61 & 3,89 & 9,26 & 1,78 & 0,56 & 0,8 & 0,16 & 99,46 \\
\hline M01-22 & Clay & 50,91 & 15,8 & 5,19 & 3,18 & 13,23 & 9,77 & 0,65 & 0,7 & 0 & 99,43 \\
\hline M01-23 & Clay & 54,19 & 16,32 & 4,85 & 3,14 & 12,16 & 7,29 & 0,76 & 0,71 & 0 & 99,43 \\
\hline M01-24 & Clay & 59,37 & 19,76 & 7,07 & 4,29 & 6,57 & 1,18 & 0,37 & 0,81 & 0,07 & 99,49 \\
\hline M01-25 & Siltstone & 65,69 & 16,86 & 4,13 & 3,29 & 5,59 & 2,2 & 0,97 & 0,89 & 0 & 99,62 \\
\hline M01-27 & Siltstone & 63,81 & 18,06 & 4,47 & 3,67 & 6,37 & 1,6 & 0,81 & 0,82 & 0 & 99,61 \\
\hline M01-28 & Clay & 59,89 & 18,63 & 7,44 & 4,21 & 6,69 & 1,26 & 0,49 & 0,86 & 0 & 99,47 \\
\hline M01-30 & Clay & 57,74 & 18,57 & 6,75 & 3,97 & 8,55 & 2,55 & 0,43 & 0,8 & 0,12 & 99,47 \\
\hline M02-1 & Clay & 43,62 & 12,43 & 4,11 & 2,52 & 17,1 & 18,28 & 0,76 & 0,55 & 0 & 99,38 \\
\hline M02-2 & Clay & 59,64 & 19,86 & 6,87 & 4,25 & 6,58 & 1,07 & 0,34 & 0,78 & 0,09 & 99,48 \\
\hline M02-3 & Clay & 61,15 & 18,36 & 6,84 & 4,04 & 6,36 & 1,44 & 0,53 & 0,86 & 0 & 99,57 \\
\hline M02-4 & Clay & 49,47 & 15,71 & 5,37 & 3,12 & 13,46 & 11,09 & 0,53 & 0,68 & 0 & 99,43 \\
\hline M02-5 & Clay & 63,61 & 17,73 & 5,06 & 3,6 & 6,11 & 1,91 & 0,72 & 0,84 & 0 & 99,56 \\
\hline M02-6 & Clay & 59,46 & 18,2 & 6,55 & 3,75 & 7,77 & 2,22 & 0,59 & 0,86 & 0 & 99,4 \\
\hline M02-8 & Clay & 25,73 & 8,84 & 2,4 & 1,7 & 23,07 & 36,49 & 0,61 & 0,32 & 0 & 99,15 \\
\hline M02-9 & Clay & 57,15 & 19,3 & 8,5 & 4,21 & 7,16 & 1,77 & 0,33 & 0,8 & 0,1 & 99,31 \\
\hline M02-10 & Clay & 39,67 & 13,6 & 5,59 & 3,27 & 16,45 & 19,77 & 0,47 & 0,6 & 0 & 99,42 \\
\hline M02-11 & Clay & 54,01 & 17,63 & 7,11 & 3,97 & 9,65 & 5,88 & 0,33 & 0,77 & 0 & 99,35 \\
\hline M02-12 & Marl & 36,31 & 12,22 & 4,72 & 2,63 & 19 & 23,69 & 0,4 & 0,38 & 0 & 99,35 \\
\hline M02-14 & Clay & 46,52 & 13,19 & 4 & 2,55 & 15,6 & 16,11 & 0,84 & 0,6 & 0 & 99,4 \\
\hline M02-14 & Siltstone & 61,08 & 17,37 & 6,14 & 4,02 & 7,95 & 1,62 & 0,58 & 0,79 & 0 & 99,56 \\
\hline M02-15 & Clay breccia & 46,75 & 15,82 & 5,45 & 3,66 & 12,74 & 13,83 & 0,35 & 0,57 & 0,07 & 99,24 \\
\hline M02-16 & Siltstone & 35,52 & 10,73 & 3,11 & 2,75 & 19,08 & 27,19 & 0,6 & 0,5 & 0 & 99,47 \\
\hline M02-17 & Marl & 35,45 & 11,32 & 3,61 & 2,68 & 20,02 & 25,5 & 0,37 & 0,44 & 0 & 99,39 \\
\hline M02-18 & Clay & 58,94 & 20,32 & 7,83 & 4,16 & 6,13 & 0,75 & 0,37 & 0,79 & 0,11 & 99,4 \\
\hline M03-5 & Clay & 49,69 & 15,32 & 5,52 & 3,43 & 11,69 & 11,79 & 0,92 & 0,77 & 0,2 & 99,34 \\
\hline M03-6 & Clay breccia & 42,67 & 13,09 & 4,27 & 2,99 & 15,38 & 19,51 & 0,74 & 0,65 & 0 & 99,29 \\
\hline M03-7 & Clay & 45,65 & 14,62 & 4,6 & 2,55 & 15 & 14,31 & 1,64 & 0,7 & 0,16 & 99,23 \\
\hline M03-8 & Clay & 50,6 & 15,44 & 5,08 & 3,02 & 12,94 & 12,08 & 0 & 0,61 & 0 & 99,76 \\
\hline M03-9 & Clay & 61,09 & 19,66 & 6,78 & 3,97 & 5,69 & 0,87 & 0,46 & 0,85 & 0,09 & 99,46 \\
\hline M03-10 & Clay & 58,45 & 19,35 & 6,47 & 4,05 & 5,97 & 4,45 & 0 & 0,81 & 0 & 99,55 \\
\hline M03-11 & Clay & 56,91 & 17,9 & 7,91 & 4,52 & 7,43 & 1,96 & 1,2 & 1,11 & 0 & 98,96 \\
\hline M03-12 & Clay & 55,2 & 18,28 & 7,82 & 3,55 & 9,06 & 4,53 & 0 & 0,74 & 0 & 99,18 \\
\hline M03-13 & Clay & 53,58 & 16,96 & 5,77 & 3,19 & 11,21 & 7,27 & 0,45 & 0,7 & 0,18 & 99,31 \\
\hline M03-14 & Clay & 60,39 & 19,14 & 7,4 & 4,18 & 5,99 & 1,07 & 0,38 & 0,86 & 0,09 & 99,5 \\
\hline
\end{tabular}


R.R. Khaziev, G.A. Krinari, N.G. Nurgalieva, G.A. Batalin and B.I. Gareev

\begin{tabular}{|c|c|c|c|c|c|c|c|c|c|c|c|}
\hline M03-15 & Clay & 58,89 & 19,62 & 8,9 & 4,07 & 5,81 & 0,86 & 0,29 & 0,82 & 0,09 & 99,35 \\
\hline M03-16 & Clay & 58,21 & 19,41 & 4,56 & 3,68 & 8,54 & 3,38 & 0,49 & 0,83 & 0 & 99,1 \\
\hline M03-17 & Clay & 49,03 & 14,68 & 5,94 & 2,95 & 13,56 & 11,64 & 0,68 & 0,73 & 0 & 99,22 \\
\hline M03-18 & Clay & 58,87 & 19,65 & 8,95 & 4,46 & 5,76 & 0,84 & 0 & 0,84 & 0,09 & 99,46 \\
\hline M03-19 & Siltstone & 64,21 & 19,33 & 4,19 & 3,6 & 5,71 & 0,97 & 0,6 & 0,86 & 0 & 99,47 \\
\hline M03-20 & Clay & 56,86 & 19,31 & 8,58 & 3,86 & 7,15 & 1,99 & 0,43 & 0,78 & 0,14 & 99,12 \\
\hline M03-21 & Clay & 50,54 & 15,46 & 5,57 & 3,47 & 12,51 & 10,75 & 0,36 & 0,68 & 0 & 99,33 \\
\hline M03-22 & Clay & 58,39 & 19,18 & 6,4 & 4,13 & 7,01 & 3,52 & 0 & 0,75 & 0 & 99,37 \\
\hline M03-23 & Clay & 56,87 & 17,79 & 5,65 & 3,75 & 8,68 & 5,5 & 0,49 & 0,71 & 0 & 99,42 \\
\hline M03-25 & Clay & 53,96 & 13,69 & 6,16 & 3,58 & 11,26 & 9,42 & 0,39 & 0,6 & 0,17 & 99,24 \\
\hline M03-26 & Clay & 26,01 & 7,94 & 2,56 & 1,64 & 27,41 & 31,8 & 0,93 & 0,24 & 0,18 & 98,71 \\
\hline M03-27 & Marl & 46,67 & 9,09 & 2,47 & 2,15 & 13,08 & 24,52 & 0,72 & 0,44 & 0 & 99,14 \\
\hline M03-28 & Clay & 47,27 & 13,62 & 5,37 & 2,98 & 14,28 & 14,28 & 0,8 & 0,61 & 0 & 99,2 \\
\hline M03-29 & Clay & 56,95 & 15,92 & 5,59 & 3,14 & 9,82 & 6,27 & 0,8 & 0,74 & 0 & 99,23 \\
\hline M05-6 & Marl & 24,49 & 5,09 & 3,27 & 1,52 & 18,46 & 44,23 & 1,11 & 0,37 & 0 & 98,54 \\
\hline M05-14 & Clay & 71,11 & 8,85 & 6,78 & 4,05 & 4,54 & 1,74 & 1,22 & 1,18 & 0 & 99,47 \\
\hline M05-26 & Clay & 62,93 & 9,96 & 6,26 & 3,97 & 6,47 & 7,62 & 0,93 & 0,91 & 0 & 99,05 \\
\hline M05-27 & Clay & 63,96 & 11,58 & 10,23 & 4,54 & 4,58 & 0,86 & 2,19 & 1,06 & 0 & 99 \\
\hline M05-32 & Clay & 71,48 & 9,93 & 4,39 & 3,82 & 4,82 & 2,84 & 0,88 & 1,09 & 0 & 99,25 \\
\hline M05-44 & Clay & 53,49 & 8,63 & 5,28 & 2,4 & 10,32 & 16,54 & 1,22 & 0,83 & 0 & 98,71 \\
\hline M05-46 & Clay & 37,49 & 7,61 & 3,37 & 2,4 & 15,22 & 30,8 & 1,13 & 0,68 & 0 & 98,7 \\
\hline M06-2 & Clay & 65,7 & 9,08 & 7,95 & 2,52 & 5,34 & 6,53 & 1,34 & 0,91 & 0 & 99,37 \\
\hline M06-3 & Siltstone & 42,93 & 7,2 & 4,37 & 2,3 & 12,22 & 28,45 & 1,06 & 0,49 & 0 & 99,02 \\
\hline M06-4 & Clay & 68,16 & 10,99 & 8,26 & 3,32 & 4,79 & 1,43 & 1,45 & 1,03 & 0 & 99,43 \\
\hline M06-6 & Siltstone & 69,98 & 11,03 & 6,56 & 3,08 & 4,97 & 1,17 & 1,72 & 1 & 0 & 99,51 \\
\hline M05-24 & Clay & 68,6 & 10,4 & 8,13 & 4,71 & 4,45 & 0,74 & 1,38 & 1 & 0 & 99,41 \\
\hline M05-25 & Clay & 63,05 & 12,74 & 8,22 & 4,31 & 5,32 & 0,89 & 2,67 & 1,14 & 0 & 98,34 \\
\hline M05-53 & Clay & 29,06 & 6,99 & 2,78 & 1,63 & 18,26 & 38,08 & 1,5 & 0,46 & 0 & 98,76 \\
\hline M05-54 & Clay & 68,03 & 11,13 & 7,25 & 4,33 & 4,91 & 0,55 & 1,89 & 0,97 & 0 & 99,06 \\
\hline M06-10 & Siltstone & 70,51 & 9,96 & 5,8 & 3,01 & 4,98 & 2,92 & 1,2 & 1,02 & 0 & 99,4 \\
\hline M06-11 & Clay & 49 & 7,4 & 6,71 & 2,65 & 9,95 & 21,67 & 1 & 0,66 & 0 & 99,04 \\
\hline M06-12 & Siltstone & 65,61 & 8,55 & 4,18 & 2,76 & 6,3 & 9,94 & 1,35 & 0,71 & 0 & 99,4 \\
\hline M06-13 & Clay & 69,37 & 10,38 & 8,67 & 3,43 & 4,11 & 1,17 & 1,23 & 0,95 & 0 & 99,31 \\
\hline M06-14 & Clay & 68,04 & 11,09 & 9,06 & 3,54 & 4,52 & 0,86 & 1,35 & 0,96 & 0 & 99,42 \\
\hline M06-15 & Marl & 32,23 & 6,32 & 3,6 & 1,54 & 15,33 & 38,62 & 0,98 & 0,43 & 0 & 99,05 \\
\hline M06-16 & Clay & 70,43 & 10,71 & 6,68 & 2,8 & 4,2 & 1,57 & 1,19 & 0,89 & 0,75 & 99,22 \\
\hline M06-17 & Sand & 79,32 & 8,28 & 3,64 & 2,11 & 3,01 & 1,2 & 1,21 & 0,69 & 0 & 99,46 \\
\hline M06-18 & Clay & 65,26 & 12,14 & 9,14 & 3,73 & 5,08 & 1,04 & 1,5 & 0,93 & 0,52 & 99,34 \\
\hline M06-19 & Clay & 66,22 & 10,91 & 8,65 & 3,83 & 5,13 & 1,45 & 1,53 & 1,03 & 0,47 & 99,22 \\
\hline M06-20 & Clay & 70,07 & 11,04 & 6,81 & 3,54 & 4,77 & 0,75 & 1,28 & 0,97 & 0,22 & 99,45 \\
\hline
\end{tabular}


Table 2. Major element composition of clayey sediments second suite (top part of table clayey sediments; other part (under the red line-boundary) - the samples from palesoils)

\begin{tabular}{|c|c|c|c|c|c|c|c|c|c|c|c|}
\hline Sample & Lithology & $\mathrm{SiO}_{2}(\%)$ & $\mathrm{Al}_{2} \mathrm{O}_{3}(\%)$ & $\mathrm{Fe}_{2} \mathrm{O}_{3}(\%)$ & $\mathrm{K}_{2} \mathrm{O}(\%)$ & $\mathrm{MgO}(\%)$ & $\mathrm{CaO}(\%)$ & $\mathrm{Na}_{2} \mathrm{O}(\%)$ & $\mathrm{TiO}_{2}(\%)$ & $\mathrm{P}_{2} \mathrm{O}_{5}(\%)$ & Total \\
\hline M07-1 & Clay & 66,65 & 10,19 & 9,4 & 4,32 & 5,24 & 0,79 & 1,12 & 1,06 & 0 & 98,75 \\
\hline M07-2 & Siltstone & 69,02 & 10,35 & 8,84 & 3,42 & 4,47 & 0,89 & 1,33 & 1,1 & 0 & 99,42 \\
\hline M07-3 & Siltstone & 36,61 & 7,06 & 2,88 & 1,74 & 11,95 & 37,68 & 1,01 & 0,45 & 0 & 99,37 \\
\hline M07-4 & Siltstone & 53,15 & 7,25 & 4,17 & 2,56 & 7,56 & 23,6 & 0,81 & 0,73 & 0 & 99,83 \\
\hline M07-13 & Clay & 46,27 & 7,1 & 2,29 & 2,28 & 8,75 & 31,03 & 0,87 & 0,61 & 0 & 99,19 \\
\hline M07-14 & Clay & 44,64 & 7,59 & 3,4 & 2,77 & 14,19 & 25,2 & 1,01 & 0,86 & 0 & 99,66 \\
\hline M07-15 & Clay & 62,55 & 9,83 & 9,22 & 4,39 & 9,86 & 1,25 & 1,06 & 1,01 & 0 & 99,16 \\
\hline M07-16 & Clay & 60,28 & 8,54 & 7,06 & 3,51 & 8,39 & 9,66 & 0,77 & 1,05 & 0 & 99,25 \\
\hline M07-18 & Clay & 62,68 & 7,3 & 8,65 & 4,22 & 11,52 & 1,37 & 1,03 & 1 & 0 & 97,77 \\
\hline M08-3 & Clay+siltstone & 72,06 & 10,74 & 5,76 & 3,69 & 4,1 & 0,73 & 1,65 & 0,72 & 0 & 99,46 \\
\hline M08-19 & Clay & 45,37 & 8,67 & 5,99 & 2,95 & 10,93 & 23,15 & 1,08 & 0,62 & 0 & 98,78 \\
\hline M08-20 & Marl & 18,72 & 4,38 & 1,76 & 1,1 & 18,54 & 53,28 & 0,99 & 0,23 & 0 & 98,99 \\
\hline M08-23 & Clay & 65,4 & 9,15 & 5,71 & 3,11 & 5,51 & 9,11 & 0,56 & 0,88 & 0 & 99,43 \\
\hline M08-25 & Clay & 26,06 & 5,2 & 2,62 & 1,43 & 15,09 & 47,32 & 0,97 & 0,31 & 0 & 99,01 \\
\hline M08-52 & Marl & 34,26 & 6,24 & 5,62 & 2,24 & 13,47 & 35,68 & 0,97 & 0,49 & 0 & 98,97 \\
\hline M08-53 & Marl & 25,83 & 4,23 & 2,85 & 1,7 & 6,45 & 57,2 & 0,6 & 0,41 & 0,05 & 99,31 \\
\hline M08-61 & Clay & 43,79 & 7,62 & 5,9 & 2,89 & 5,44 & 31,95 & 0,84 & 0,7 & 0 & 99,13 \\
\hline M08-62 & Clay & 63,77 & 9,94 & 6,73 & 3,77 & 4,66 & 7,96 & 0,94 & 0,95 & 0,44 & 99,14 \\
\hline M08-74 & Marl & 45,38 & 7,62 & 4,86 & 2,32 & 9,3 & 27,59 & 1,03 & 0,62 & 0 & 98,71 \\
\hline M08-75 & Marl & 34,26 & 6,24 & 5,62 & 2,24 & 13,47 & 35,68 & 0,97 & 0,49 & 0 & 98,97 \\
\hline
\end{tabular}

Table 3. Major element composition of clayey sediments third suite

\begin{tabular}{|c|c|c|c|c|c|c|c|c|c|c|c|}
\hline Sample & Lithology & $\mathrm{SiO}_{2}(\%)$ & $\mathrm{Al}_{2} \mathrm{O}_{3}(\%)$ & $\mathrm{Fe}_{2} \mathrm{O}_{3}(\%)$ & $\mathrm{K}_{2} \mathrm{O}(\%)$ & MgO (\%) & $\mathrm{CaO}(\%)$ & $\mathrm{Na}_{2} \mathrm{O}(\%)$ & $\mathrm{TiO}_{2}(\%)$ & $\mathrm{P}_{2} \mathrm{O}_{5}(\%)$ & Total \\
\hline M07-5 & Clay & 55,48 & 11,04 & 4,97 & 3,09 & 4,7 & 17,91 & 1,38 & 0,71 & 0 & 99,29 \\
\hline M07-6 & Clay & 61,33 & 11,14 & 6,37 & 3,38 & 3,89 & 10,28 & 1,9 & 0,88 & 0 & 99,16 \\
\hline M07-7 & Clay & 58,91 & 11,03 & 4,56 & 3,65 & 4,23 & 14,95 & 1,41 & 0,95 & 0 & 99,69 \\
\hline M07-8 & Clay & 66,01 & 11,63 & 2,23 & 1,51 & 4,02 & 12,61 & 1,49 & 0,37 & 0 & 99,88 \\
\hline M07-9 & Clay & 58,41 & 10,95 & 5,69 & 3,24 & 4,74 & 14,85 & 1,08 & 0,76 & 0 & 99,71 \\
\hline M07-10 & Clay & 65,83 & 11,8 & 2,46 & 1,67 & 3,87 & 11,52 & 1,43 & 0,45 & 0 & 99,02 \\
\hline M07-11 & Clay & 67,95 & 9,12 & 6,97 & 3,75 & 4,52 & 4,38 & 1,61 & 1,03 & 0 & 99,32 \\
\hline M07-12 & Clay & 65,01 & 5,25 & 7,94 & 3,3 & 13,27 & 0,93 & 0,9 & 1,12 & 0 & 97,72 \\
\hline M08-5 & Clay & 69,52 & 10,93 & 7,98 & 3,47 & 4,22 & 0,71 & 1,51 & 0,83 & 0,22 & 99,39 \\
\hline M08-6 & Clay & 68,17 & 10,98 & 8,74 & 3,37 & 4,36 & 0,81 & 1,74 & 0,89 & 0,34 & 99,41 \\
\hline M08-7 & Clay & 68,42 & 10,9 & 7,8 & 3,27 & 4,44 & 1,05 & 1,92 & 1,1 & 0,37 & 99,27 \\
\hline M08-27 & Clay & 48,85 & 7,03 & 4,42 & 2,51 & 9,61 & 25,13 & 0,92 & 0,64 & 0 & 99,1 \\
\hline M08-30 & Clay & 72,2 & 10,93 & 7,57 & 3,24 & 3,52 & 0,96 & 0,17 & 1,04 & 0 & 99,62 \\
\hline M08-31 & Clay & 60,31 & 9,02 & 7,05 & 3,04 & 7,22 & 10,74 & 1 & 0,94 & 0 & 99,32 \\
\hline M08-32 & Clay & 46,07 & 9,27 & 5,65 & 2,92 & 10,5 & 23,01 & 0,89 & 0,7 & 0 & 99 \\
\hline M08-37 & Clay & 70,1 & 10,86 & 7,55 & 3,14 & 4,52 & 1,19 & 1,26 & 0,9 & 0 & 99,51 \\
\hline M08-39 & Marl & 40,64 & 6,47 & 5,43 & 2,8 & 7,03 & 35,56 & 0,83 & 0,58 & 0 & 99,35 \\
\hline
\end{tabular}


R.R. Khaziev, G.A. Krinari, N.G. Nurgalieva, G.A. Batalin and B.I. Gareev

\begin{tabular}{|l|c|c|c|c|c|c|c|c|c|c|c|}
\hline M08-41 & Clay+siltstone & 59,89 & 9,56 & 9,95 & 2,54 & 4,48 & 10,45 & 1,32 & 1,02 & 0 & 99,2 \\
\hline M08-44 & Clay & 64,38 & 9,92 & 9,08 & 2,75 & 4,94 & 5,35 & 1,48 & 1,04 & 0,3 & 99,24 \\
\hline M08-45 & Clay+siltstone & 67,29 & 9,56 & 5,3 & 2,73 & 4,37 & 8,94 & 0 & 1 & 0 & 99,19 \\
\hline M08-46 & Clay+siltstone & 65,41 & 9,97 & 5,25 & 3 & 4,98 & 8,55 & 1 & 1,04 & 0 & 99,2 \\
\hline M08-47 & Clay & 62,55 & 11,09 & 10,85 & 3,85 & 5,02 & 3,2 & 1,26 & 1,01 & 0,41 & 99,22 \\
\hline M08-55 & Clay & 48,86 & 6,76 & 4,64 & 2,6 & 6,79 & 27,93 & 0,8 & 0,64 & 0 & 99 \\
\hline M08-56 & Clay & 65,39 & 10,4 & 6,96 & 2,64 & 5,27 & 5,84 & 0,89 & 1,35 & 0 & 98,74 \\
\hline M08-57 & Clay+siltstone & 64,88 & 10,64 & 11,34 & 2,7 & 4,75 & 1,93 & 1,63 & 1,1 & 0,35 & 99,34 \\
\hline M08-59 & Clay & 64,61 & 9,78 & 8,58 & 2,89 & 4,76 & 6,53 & 1,19 & 1 & 0 & 99,35 \\
\hline M08-63 & Clay & 65,37 & 10,3 & 8,68 & 2,97 & 5,01 & 4,76 & 1,27 & 1,06 & 0 & 99,42 \\
\hline M08-64 & Clay & 63,91 & 10,25 & 8,11 & 2,62 & 4,92 & 7,21 & 0,95 & 1,14 & 0 & 99,11 \\
\hline M08-65 & Clay & 65,34 & 11,19 & 8,96 & 2,87 & 5,28 & 2,01 & 2,02 & 1,1 & 0,38 & 99,14 \\
\hline M08-66 & Clay+siltstone & 70,81 & 9,74 & 8,36 & 3,2 & 4,01 & 0,89 & 1,56 & 0,91 & 0 & 99,47 \\
\hline
\end{tabular}

Table 3. Major element composition of paleosoils of third suite

\begin{tabular}{|c|c|c|c|c|c|c|c|c|c|c|c|}
\hline M11-1 & Clay & 66,1 & 11,53 & 9,91 & 2,72 & 4,57 & 1,18 & 1,89 & 1,18 & 0 & 99,08 \\
\hline M13-10 & Clay & 36,6 & 6,3 & 4,03 & 2,33 & 5,16 & 43,52 & 0,81 & 0,56 & 0 & 99,31 \\
\hline M14-4 & Clay & 51,49 & 9,03 & 10,41 & 3,66 & 7,99 & 14,25 & 0,88 & 0,89 & 0,31 & 98,91 \\
\hline M14-24 & Clay breccia & 45,05 & 9,05 & 6,21 & 2,27 & 10,07 & 24,2 & 1,04 & 0,72 & 0 & 98,61 \\
\hline M15-13 & Marl & 41,65 & 7,69 & 5,28 & 2,19 & 11,52 & 28,68 & 0,93 & 0,67 & 0 & 98,61 \\
\hline M15-38 & Clay+siltstone & 63,62 & 10,58 & 10,85 & 2,7 & 4,93 & 4,33 & 1,18 & 1,05 & 0 & 99,24 \\
\hline M16-12 & Sandstone & 59,61 & 7,05 & 4,81 & 1,68 & 2,78 & 21,15 & 1,07 & 0,65 & 0,21 & 99,01 \\
\hline M16-15 & Clay+siltstone & 71,09 & 11,12 & 5,87 & 2,7 & 4,28 & 2,03 & 1,2 & 1,05 & 0 & 99,34 \\
\hline M16-20 & Siltstone & 68,12 & 10,25 & 6,82 & 2,51 & 4,71 & 4,76 & 1,07 & 1,05 & 0 & 99,29 \\
\hline M16-22 & Clay+siltstone & 66,38 & 10,82 & 9,64 & 2,6 & 4,46 & 2,98 & 1,48 & 1,03 & 0 & 99,39 \\
\hline M16-26 & Clay & 54,08 & 8,68 & 6,09 & 2,65 & 8,2 & 17,53 & 0,68 & 0,94 & 0 & 98,85 \\
\hline M16-27 & Clay & 53,85 & 8,55 & 7,75 & 2,63 & 8,05 & 16,14 & 1,1 & 0,82 & 0 & 98,89 \\
\hline M16-28 & Marl & 35,71 & 6,63 & 3,8 & 2 & 13,07 & 37,22 & 0 & 0,62 & 0 & 99,05 \\
\hline M16-30 & Siltstone & 56,31 & 8,26 & 5,83 & 2,63 & 6,88 & 17,59 & 0,69 & 0,89 & 0 & 99,08 \\
\hline M16-34 & Clay+siltstone & 68,3 & 10,26 & 8,09 & 3,56 & 4,02 & 2,81 & 1,11 & 1,1 & 0 & 99,25 \\
\hline
\end{tabular}

Table 4. Major element composition of clayey sediments fourth suite

\begin{tabular}{|c|c|c|c|c|c|c|c|c|c|c|c|}
\hline Sample & Lithology & $\mathrm{SiO}_{2}(\%)$ & $\mathrm{Al}_{2} \mathrm{O}_{3}(\%)$ & $\mathrm{Fe}_{2} \mathrm{O}_{3}(\%)$ & $\mathrm{K}_{2} \mathrm{O}(\%)$ & $\mathrm{MgO}(\%)$ & $\mathrm{CaO}(\%)$ & $\mathrm{Na}_{2} \mathrm{O}_{(\%)}$ & $\mathrm{TiO}_{2}(\%)$ & $\mathrm{P}_{2} \mathrm{O}_{5}(\%)$ & $\mathrm{Total}^{\prime}$ \\
\hline M12-3 & Clay & 59,24 & 9,32 & 9,97 & 2,48 & 7,03 & 8,36 & 1,43 & 1,03 & 0 & 98,86 \\
\hline M12-4 & Siltstone & 63,44 & 8,89 & 4,89 & 2,35 & 5,76 & 11,87 & 0,82 & 1,05 & 0 & 99,07 \\
\hline M12-6 & Clay & 48,86 & 6,76 & 4,64 & 2,6 & 6,79 & 27,93 & 0,8 & 0,64 & 0 & 99,02 \\
\hline M12-8 & Clay & 49,14 & 10,17 & 7,55 & 3,44 & 9,39 & 17,12 & 1,06 & 0,82 & 0 & 98,69 \\
\hline M12-10 & Clay & 66,43 & 14,23 & 8,96 & 4,08 & 3,47 & 1,11 & 0 & 1,01 & 0 & 99,29 \\
\hline M13-2 & Clay & 59,51 & 9,04 & 8,59 & 2,96 & 6,76 & 10,17 & 1,1 & 0,94 & 0 & 99,07 \\
\hline M14-12 & Clay & 63,54 & 10,35 & 9,68 & 2,7 & 5,38 & 4,71 & 1,53 & 1,13 & 0 & 99,02 \\
\hline M14-13 & Clay+siltstone & 64,7 & 10,37 & 8,95 & 2,78 & 4,53 & 5,09 & 1,84 & 1,04 & 0 & 99,3 \\
\hline M14-17 & Clay+siltstone & 64,88 & 10,64 & 11,34 & 2,7 & 4,75 & 1,93 & 1,63 & 1,1 & 0,35 & 99,32 \\
\hline M14-20 & Clay & 67,85 & 11,33 & 6,23 & 2,79 & 4,94 & 3,77 & 1,28 & 0,97 & 0 & 99,16 \\
\hline
\end{tabular}




\begin{tabular}{|l|c|c|c|c|c|c|c|c|c|c|c|}
\hline M14-21 & Clay & 65,27 & 10,76 & 10,55 & 2,76 & 4,68 & 2,28 & 1,81 & 1,04 & 0 & 99,15 \\
\hline M14-22 & Siltstone & 67,05 & 9,72 & 4,93 & 2,38 & 4,53 & 9,43 & 0 & 1,14 & 0 & 99,18 \\
\hline M14-23 & Clay+siltstone & 62,6 & 10,53 & 9,98 & 2,38 & 5,14 & 6,41 & 1,09 & 1,04 & 0 & 99,17 \\
\hline M15-21 & Clay & 67,54 & 10,59 & 8,34 & 3,85 & 4,45 & 1,64 & 1,35 & 1,03 & 0,54 & 99,33 \\
\hline M15-25 & Clay & 65,98 & 10,59 & 11,35 & 2,97 & 4,38 & 1,14 & 1,36 & 1,13 & 0,3 & 99,2 \\
\hline M15-27 & Clay+siltstone & 65,78 & 11,61 & 9,8 & 2,68 & 4,6 & 1,15 & 1,99 & 1,17 & 0,3 & 99,08 \\
\hline M15-29 & Clay & 65,5 & 10,7 & 11,23 & 2,67 & 4,58 & 1,11 & 2 & 1,13 & 0,32 & 99,24 \\
\hline M15-32 & Clay+siltstone & 66,1 & 12,23 & 8,45 & 2,54 & 4,92 & 1,46 & 2,22 & 1,15 & 0 & 99,07 \\
\hline M15-35 & Clay+siltstone & 64,94 & 11,12 & 9,07 & 2,82 & 4,42 & 4,31 & 1,28 & 1,14 & 0 & 99,1 \\
\hline M15-40 & Clay+siltstone & 66,16 & 11,04 & 7,71 & 2,36 & 5,01 & 4,96 & 0,91 & 1,02 & 0 & 99,17 \\
\hline M15-44 & Clay & 38,35 & 7,53 & 6,83 & 2,82 & 9,99 & 31,39 & 0 & 0,91 & 0 & 97,82 \\
\hline
\end{tabular}

Table 4. Major element composition of paleosoils of fourth suite

\begin{tabular}{|c|c|c|c|c|c|c|c|c|c|c|}
\hline Sample & Lithology & $\mathrm{SiO}_{2}(\%)$ & $\mathrm{Al}_{2} \mathrm{O}_{3}(\%)$ & $\mathrm{Fe}_{2} \mathrm{O}_{3}(\%)$ & $\mathrm{K}_{2} \mathrm{O}(\%)$ & $\mathrm{MgO}(\%)$ & $\mathrm{CaO}(\%)$ & $\mathrm{Na}_{2} \mathrm{O}(\%)$ & $\mathrm{TiO}_{2}(\%)$ & Total \\
\hline M17-2 & Clay & 49,14 & 10,17 & 7,55 & 3,44 & 9,39 & 17,12 & 1,06 & 0,82 & 98,69 \\
\hline M17-3 & Sandstone & 67,05 & 9,72 & 4,93 & 2,38 & 4,53 & 9,43 & 0 & 1,14 & 99,18 \\
\hline M17-4 & Clay+Siltstone & 62,07 & 8 & 6,34 & 2,67 & 4,66 & 13,54 & 1 & 0,96 & 99,24 \\
\hline
\end{tabular}

Table 5. Major element composition of clayey sediments fifth suite (Note: the sample marked in red - paleosoil level)

XRF spectrometry is an analytical laboratory technique used to detect the presence of specified elements in a sample material and subsequently determine the concentration of those elements present.

Bulk chemical analysis of samples was carried out using Energy Dispersive X-Ray Fluorescence method (EDXRF) on a fully-automated S2 RANGER (Bruker ASX) spectrometer having Pd- tube as X-ray source and silicon drift detector.

Preparing of sample for analysis included its crushing in mill for 5 minutes to a particle size of about 40-50 $\mu \mathrm{m}$; compaction with boric acid in a press with a force of 200 $\mathrm{kN}$ to get a tablet with a very smooth surface. Next, the tablet was placed in the instrument for analysis.

The result can be received as a percentage of the components or in terms of their oxides in a concentration range from $100 \%$ to ppm- level.

Forty five components were determined by XRF. Nine major components $\left(\mathrm{SiO}_{2}, \mathrm{Al}_{2} \mathrm{O}_{3}, \mathrm{MgO}, \mathrm{CaO}, \mathrm{Fe}_{2} \mathrm{O}_{3}, \mathrm{~K}_{2} \mathrm{O}\right.$, $\mathrm{Na}_{2} \mathrm{O}, \mathrm{TiO}_{2}, \mathrm{P}_{2} \mathrm{O}_{5}, \mathrm{SrO}$ ) were selected for clayey sediments genesis reconstruction.

\section{Results and Discussion}

On diagrams $\mathrm{K}_{2} \mathrm{O} / \mathrm{Na}_{2} \mathrm{O}$ and $\mathrm{SiO}_{2} / \mathrm{Al}_{2} \mathrm{O}_{3} \frac{16,17}{\text { (Figure 4) }}$ the sediments can be attributed to the passive continental margin with the possible presence of pyroclastic material. The sediments of all five suites were formed in the basin with a single, relatively stable distributive province, proposed in $\frac{18}{18}$.

As it can be seen from Figure $5 \frac{19,20}{2}$, weathering crust of the Urals' mafic can be the sources for clayey sediments. They usually produce glandular smectite (close to nontronite) and $\mathrm{Fe}-\mathrm{Mg}$-chlorite in the form of stable minerals.

On spider-diagrams ${ }^{21}$ normalized to PAAS (Figure 6) one can find that the maximum fluctuation value of this parameter in the samples of both suites, as well as paleosoils levels occur in oxides of calcium and magnesium, which is easily explained by precipitation or dissolution of carbonates. A high content of phosphorus oxide is outlined. It is related to sedimentary clays in the pedogenous levels of the second, third and fourth suites.

According to the study of clayey sediments performedby previous researchers, the clayey sediments of the five suites are mainly of illite-smectite composition; silt grains are composed of quartz, plagioclase, K-feldspar and muscovite plates. On the diagram A - CN - K, which was constructed according to $\frac{22}{2}$, these conclusions are confirmed (Figure 7). As kaolinite and minerals of free aluminum are not typical for arid climate and 


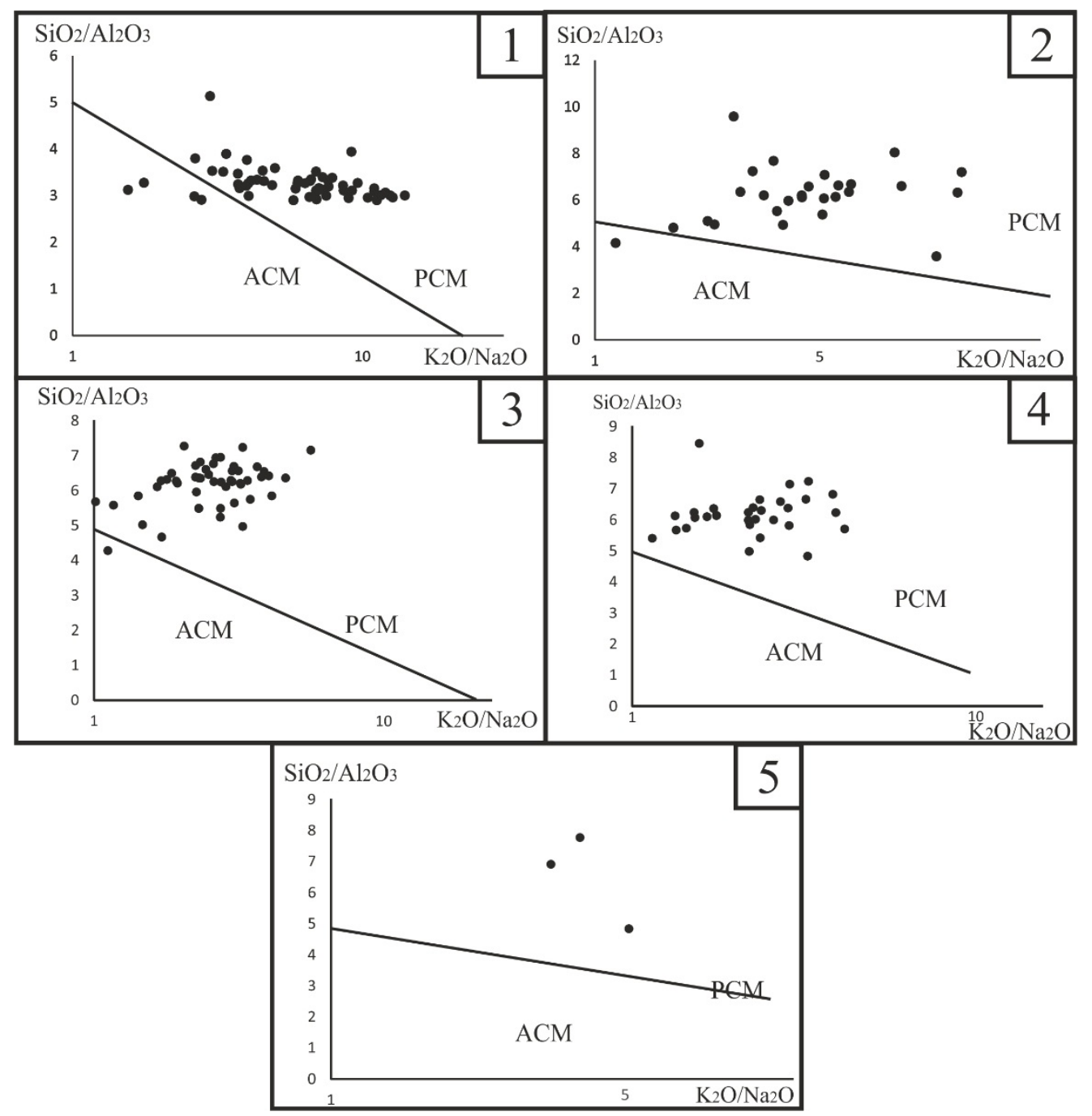

Figure 4. Relationship diagram of $\mathrm{K}_{2} \mathrm{O} / \mathrm{Na}_{2} \mathrm{O}$ and $\mathrm{SiO}_{2} / \mathrm{Al}_{2} \mathrm{O}_{3}$ for clayey rocks: 1) first 2) second 3) third; 4) fourth 5) fifth suites. Note: diagrams for sediments 2-5 suites is built with regard paleosoils levels. The separation line on the chart between the active continental margin (active continental margin - ACM) and passive continental margin (passive continental margin - PCM)-build according to ${ }^{16,17}$.

have never been recorded in the red-colored Permian rocks, the presences of $\mathrm{Mg}$ and $\mathrm{Fe}$ in the calculations were not taken into consideration; the dependence on Figure 7 largely reflects changes in the content of smectite and illite. A similar pattern of points distribution in the identical diagrams can be observed in the mudstone formation "Karak" in Northern Pakistan $\frac{23}{\text {, }}$ and is interpreted as a result of low-temperature smectite illitization.

Such kind of dependence may also be affected by the impurity of allogenic potassium feldspar, which can explain the spread of values on the local parts of the curve (Figure. 3, 6). But this is not an essential factor for the rest of the outcrop. Otherwise, the ratio of $\mathrm{SiO}_{2} /$ $\mathrm{Al}_{2} \mathrm{O}_{3}$ to $\mathrm{K}_{2} \mathrm{O} / \mathrm{Na}_{2} \mathrm{O}$ (Figure. 4,5) could not remain linear for the prevailing part of objects. Thus, there can be observed a process of direct low-temperature smectite illitization in poorly lithified sediment due to the abrupt change of the magnitude and sign of the redox potential of the mud water at the change of biotic assemblage.

The intensity of the process in the environments of arid climate is determined by hydrochemistry in sediment, it is to a certain extent defined by volumes of freshwater runoff in the basin for a certain period of time. Thus, in the outcrop there is expected variation in the proportion 


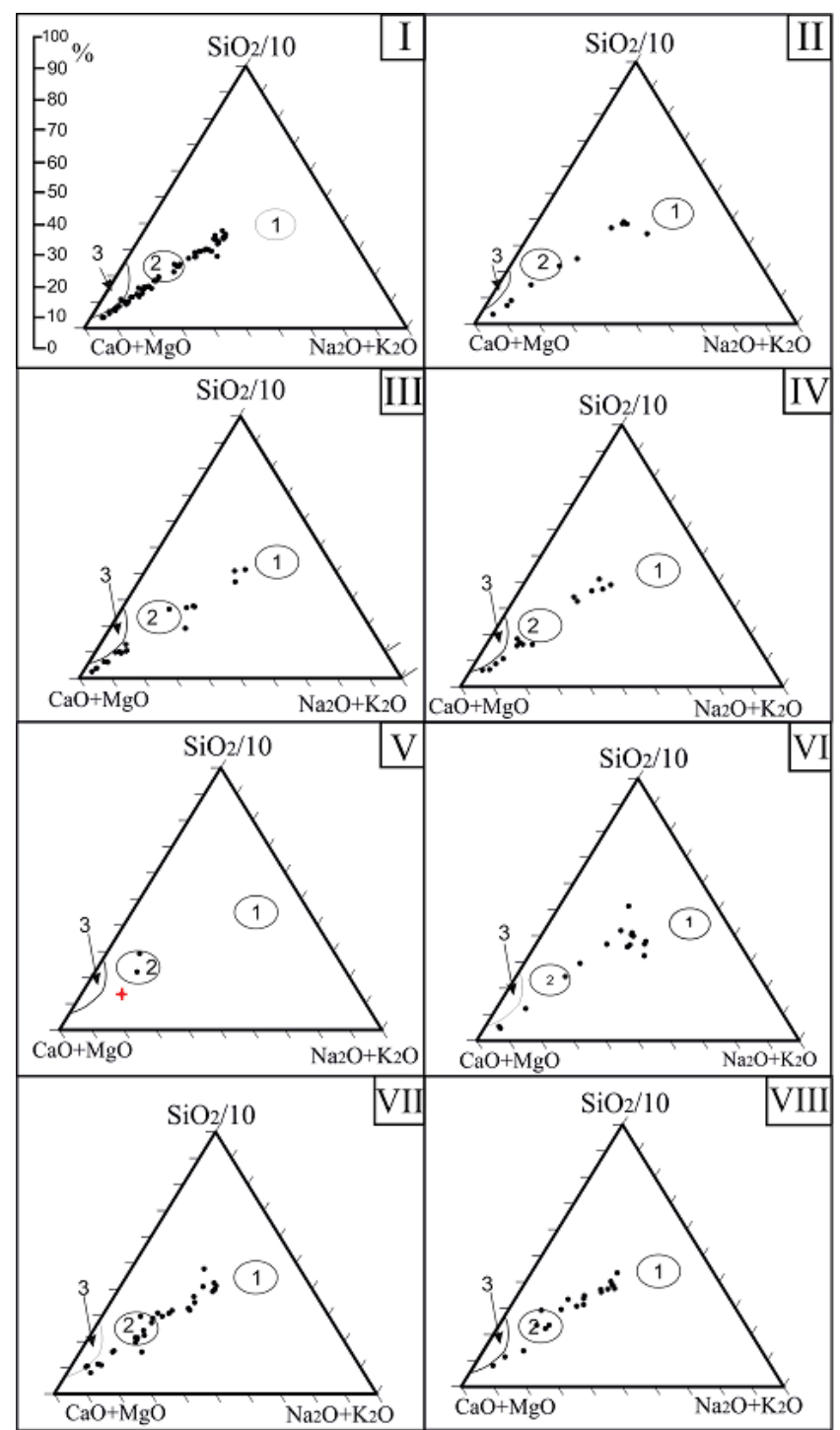

Figure 5. The diagrams constructed according to 19,20 to reconstruction of sources of demolition for clayey sediments: I) the first; II) the second; III) the third IV) the fourth V) the fifth suites. For the paleosoil levels: VI) the second VII) the third III) by the fourth it is twisted. Demolition sources: 1 granites, 2 - basalts, 3 - ultramafits the Note: on the chart $\mathrm{V}$ the red point noted a sample from paleosoil.

of mica components in the mixed-phase illite-smectite, changes in the amount of $\mathrm{K}^{+}$, fixed by packages of a newly formed mica synchronously with partial replacement of $\mathrm{Si}^{4+}$ to $\mathrm{Al}^{3+}$ in tetrahedral-meshed layers of $2: 1$. In such conditions products of biochemical decomposition of feldspars become the source of free aluminum. Similar phenomena were observed in the recent sediments of lake Hubsugul, Mongolia ${ }^{24}$.

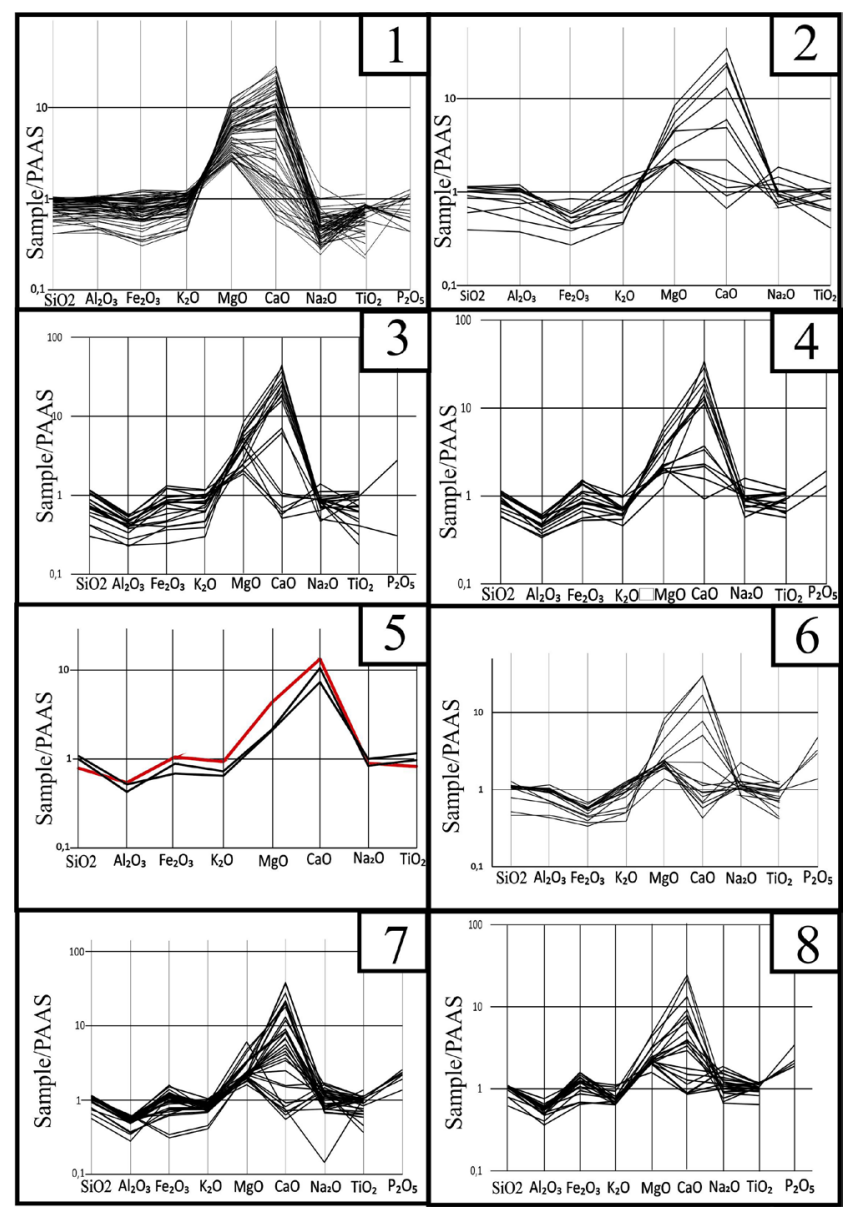

Figure 6. Spider-diagrams normalized to PAAS for clayey sediments: I) the first; II) the second; III) the third IV) the fourth V) the fifth suites. For the pedogenous levels: VI) the second VII) the third VIII) by the fourth suites. Note: Red line in diagram №5 for sample from palesoil level in the fihth suite. Data of chemical composition PAAS were taken from ${ }^{21}$.

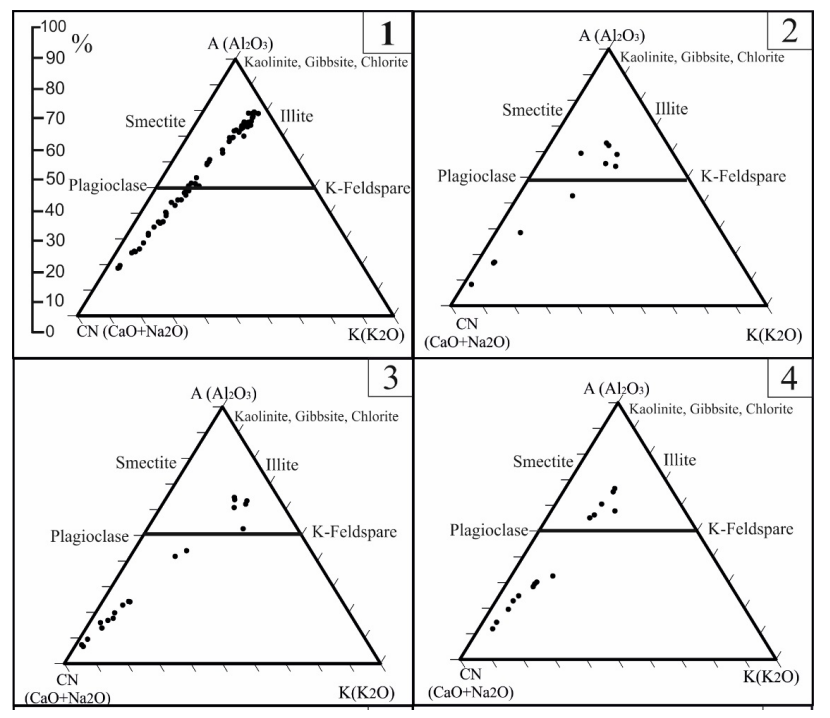




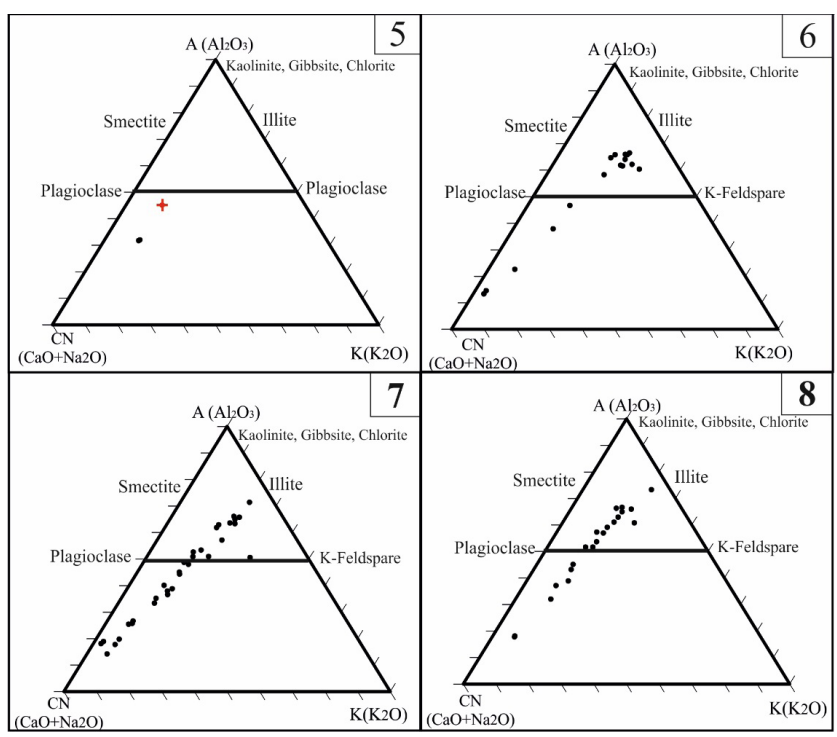

Figure 7. Plots in the $\mathrm{A}-\mathrm{CN}-\mathrm{K}$ diagram ${ }^{22}$ for clayey sediments: 1-first, 2-second, 3-third, 4-fourth, 5-fifth suits; for paleosols of 6-second, 7-third, 8-fourth suits. (Note: red point in the diagram №5 - sample from paleosol level in fifth suite.

\section{Conclusions}

The Middle-Late Permian basin at the east of Russian plate was conditioned by specific geochemical environments. A very low temperature transformation of smectite to other layered structures was very seldom recorded and concerned only illitization. The possibility of formation of such structures by the type 2: 1: 1 has not been previously detected. For the implementation of all the recorded phenomena the following conditions must be performed.

Firstly, there must be a clear arid climate, wherein in the sedimentation basin particulate material is demolished, which is non-equilibrium with water in mud and organic material preserved from oxidation of the continent, providing intensive development of microflora in poorly consolidated sediments ${ }^{25}$.

Secondly, in the sources of the ablation during the hypergenesis there must prevail rock-forming minerals from the group of smectites, which will prevail in the precipitate as well ${ }^{26}$.

Third, there must be performed certain conditions to ensure sharp and rapid changes of hydrochemical parameters of the basin. These include periodic restoration of connection with the global ocean or a temporary increase in the concentration of $\mathrm{Mg}^{2+}$ due to the intensification of evaporation of any inflow of water from the underlying rocks formed in evaporite environments $\frac{27,28}{}$.
It is evident that for most sedimentary rocks the sharing of all these conditions, which were fully realized in the investigated object, are the exception rather than the rule. Usually smectite illitization occurs in rocks that are under deep diagenesis as the result of increase of reservoir temperatures and pressures ${ }^{29}$.

\section{Acknowledgement}

The research was supported by Russian Foundation for Basic Research (Project No.15-55-10007). The financial support of the RFBR scientific research projects under 15-55-10007, 16-05-00706 project numbers and Russian Government Program of Competitive Growth of Kazan Federal University is gratefully acknowledged.

\section{References}

1. Forsh NN. On stratigraphic partition and correlation of the sections of the Tatarian stage in the east of the Russian Platform based on the complex of lithologic and stratigraphic, paleomagnetic, and paleontologic data. Tr. Vses. Neft. Nauchno-Issled. Geologorazved. Inst., Leningrad, Gostoptekhizdat;1963. p.175-211.

2. Gusev AK. Tatarian stage. Stratotypes and basic sections of the Upper Permian in theVolga region and Prikamye. Kazan, Ekotsentr;1996.p.131-41.

3. Geology of the Prikazansky Region. Guide to the Grounds of Geological Practices.Shevelev AI, editors, Kazan, Novoe Znanie; 2007. p. 208.

4. Geology of Tatarstan: Stratigraphy and Tectonics. Burov BV, editor, Moscow, GEOS; 2003. p.403.

5. Shinkarev A.A. Structural and phase heterogeneity of organic smectites in natural objects. Extended Abstract of C and. Geol.-Mineral. Sci. Diss. Moscow; 2011.p.24.

6. Krinari GA, Khramchenkov MG. Smectiteillitization of asbiotic/abiotic process. Dokl.Ross. Akad. Nauk. 2005; 403(5):664-9.

7. Kashik SA. Development of mineral zonesinwastemantels. Novosibirsk,Nauka;1989. p.161.

8. VeldeB, Meunier A. The Origin of Clay Minerals in Soils and Weathered Rocks. Berlin, Heidelberg, Springer; 2008. p.426.

9. Arefiev MP, Silantiev VV. Sedimento logical and geochemical criteriafor allocation recurrence in the reference section of Urzhumian and Severodvinsk stages "Monastirskii Ravine" (Kazan Volga Region). Material y10-goUral'skogolitolog. soveshch. Virtual 'nyeireal' nyelitologicheskiemodelis. Proceedings $10^{\text {th }}$ Ural Lithol.Meet. Virtual and Real 
Lithologic Models. Yekaterinburg, IGG Ural. Otd. Ross. Akad. Nauk.2014;157(2):18-20.

10. Ignatiev VI. The Tatarian Stage of the Central and Eastern Regions of the Russian Platform. Kazan, Izd.Kazan. Univ.;1962. p.337.

11. Miropolskiy LM. Topogeochemical Research on the Permian Deposits in Tataria. Moscow, Izd. Akad. Nauk SSSR; 1956. p.264.

12. Sementovskiy YV. Conditions of Formation of Mineral Deposits during the Late Permian Erain the East of the Russian Platform. Kazan, Tatar. Kn. Izd.; 1973.p. 255.

13. Silantiev VV, Esin DN. The reference section of the Tatarian stage in the Monastyrskii Ravine. Vestn.Mosk.Univ.,Ser. Geol.1993; 4(4):38-48.

14. Krinari GA. Paleogeography of the Tatarian basin around the parastratotype based on the Lithological and mineralogical data. Stratotipy i opornye razrezy verkhnei permi Prikazanskogo raiona: Materialyk Mezhdunar. Simpoziumu. Verkhnepermskie stratotipy Povolzhya. Stratotypes and Reference Sections of the Upper Permian in the Prikazansky Region: Proc. Int. Symp. Upper Permian Stratotypes of the Volga Region. Moscow, GEOS; 1998. p. 80-4.

15. Nurgalieva NG, Khaziev RR, Gareev BI, Batalin GA. Urzhumian stage in geochemical variations. ARPN :: Journal of Engineering and Applied Sciences. 2014; 9(5):757-64.

16. Roser BP, Korsch RJ. Determination of tectonic setting of sand stone-mudstonesuitesusingSiO2 content and $\mathrm{K} 2 \mathrm{O} /$ $\mathrm{Na} 2 \mathrm{O}$ ratio. The Journal of Geology. 1986; 94(5):635-50.

17. GhoshS,SarkarS.Geochemistryof Permo-Triassicmudstone of the Satpura Gondwanabasin, CentralIndia: Clues forprovenance. Chemical Geology.2010; 277(1-2):78-100.

18. Tikhvinskaya EI. Fundamentals of paleogeography of the east of the Russian Platform in the Upper Permian time. Dokl. Akad. Nauk SSSR. 1949; 64(2):233-6.

19. Letnikova EF. Geochemical types of carbonate deposits of the southern framing of the Siberian Platform. Extended Abstract of Cand. Geol.-Mineral. Sci. Diss. Novosibirsk; 2008. p.39.

20. Taylor SR, Mak'Lennan SM. Continental crust: Mineral composition and evolution. M.: Mir; 1988. p.384.
21. Nagarajan R, Madhavaraju J, Nagendra R, ArmstrongAltrin JS, Moutte J. Geochemistry of Neoproterozoicshales of the Rabanpalli Formation, BhimaBasin, Northern Karnataka, Southern India: Implications for provenanceand paleoredox conditions. Revista Mexicana de Ciencias Geológicas.2007; 24(2):150-60.

22. Nesbitt HW. Petrogenesis of siliciclastic sediments and sedimentary rocks. Geochemistry of Sediments and Sedimentary Rocks: Evolutionary Considerations to Mineral-Deposit-Forming Environ-ments. Lentz RD. Saint-Jones, Canada, Geol. Assoc. Canada GEO text. 2003; 4(8):39-51.

23. Saleemi AA, Ahmed Z. Mineral and chemical composition of Karak mudstone, Kohat Plateau, Pakistan: Implications for smectite illitization and provenance/ Sedimentary Geology. 2000; 130(3-4):229-47.

24. Solotchina EP. Structural typomorphism of clay minerals of sedimentary sections and waste mantels. Novosibirsk, Geo; 2009. p.234.

25. Shahraki I, Harami RM, Mahboubi A, Davoudjahani. Facies analysis, depositional environment and cyclostratigraphy of the lower permian deposits chili formation in the Kalmard Block, East Central Iran (godar-e-gachal Section). Indian Journal of Science and Technology. 2014 Jan; 7(10). DOI:10.17485/ijst/2014/v7i10/50885.

26. Mood SH, Shahraki J, Jami M, Javdan MJ, Nastooh M. Microfacies analysis, environmental reconstruction and determination of sea-level fluctuations in permian deposits of the Kalmard Area, Khan Gorup (Kuh-e-Ahaki Section). Indian Journal of Science and Technology. 2015 Feb; 8(S3). DOI: 10.17485/ijst/2015/v8iS3/60475.

27. Jayarin RJ, Ravi T. Measurement of surface ozone and its precursor NOx over Urban and Rural locations in Kannur - A tropical coastal site in India. Indian Journal of Science and Technology. 2010 Dec; 3(12).DOI:10.17485/ijst/2010/ v3i12/29862.

28. Gavva VK, Denisov AG, Arbuzova DP. Monitoring of innovative technologies and projects in the field of improved industrial energy efficiency and the industrial implementation of new energy sources aimed at sustainable development of the Northern (Arctic) Russian Regions. Indian Journal of Science and Technology. 2016 Mar; 9(12). DOI:10.17485/ijst/2016/v9i12/89518.

29. Clauer N, Williams LB, Fallick AE. Genesis of nanometric illite crystals elucidated by light-element (hydrogen, lithium, boron and oxygen) isotope tracing, and $\mathrm{K}-\mathrm{Ar}$ and Rb-Sr dating. Chemical Geology.2014; 383:26-50. 